\title{
Production of Scintillating Fiber Modules for high resolution tracking devices
}

\section{Thomas Kirn*, Roman Greim, Wacek Karpinski, Stefan Schael, Tobias Schateikis, Georg Schwering, Arndt Schultz von Dratzig and Michael Wlochal}

Rheinisch-Westfaelische Tech. Hoch. (DE)

E-mail: kirn@physik.rwth-aachen.de, greimephysik.rwth-aachen.de

karpinsk@physik.rwth-aachen.de schael@physik.rwth-aachen.de,

schateikis@physik.rwth-aachen.del schwerin@physik.rwth-aachen.de,

svdephysik.rwth-aachen.de wlochalephysik.rwth-aachen.de

In high energy physics experiments tracking detectors consisting of scintillating fibres readout by linear arrays of silicon photomultiplier have become a competitive alternative to silicon strip detectors.

The modules produced at the I. Physics Institute of RWTH Aachen University are made out of mats of $0.25 \mathrm{~mm}$ diameter scintillating fibres. Mats with different amount of layers and with a length of $0.3 \mathrm{~m}$ up to $3 \mathrm{~m}$ have been produced. A spatial resolution of $0.05 \mathrm{~mm}$ was achieved with prototypes and experiments using these scintillating fibre modules as tracking detectors.

The established process of scintillating fibre module production and quality control measurements will be presented. As examples of use the balloon experiment PERDaix, a myontomograph and the new Beam Gas Vertex (BGV) detector will be also described shortly.

Technology and Instrumentation in Particle Physics 2014,

2-6 June, 2014

Amsterdam, the Netherlands

* Speaker. 


\section{Introduction}

A new type of scintillating fibre tracker has been developed by using tightly packed fibre mats by using fibres with a diameter of $0.25 \mathrm{~mm}$ and for the readout silicon photomultipliers (SiPMs) as photodetectors to achieve a spatial resolution of $0.05 \mathrm{~mm}$. SiPMs have a high intrinsic gain of $10^{5}-10^{6}$ and photodetection efficiencies of $40 \%$ and can be operated at room temperature. They are insensitiv to magnetic fields, require operating voltages of $20-80 \mathrm{~V}$ and have compact dimensions so that they can be coupled directly to the scintillating fibres.

\section{Scintillating Fibre Mats for tracking devices}

Kuraray SCSF-78MJ fibres were chosen for the tracker because their peak emission wavelength at $450 \mathrm{~nm}$ matched the peak sensitivity of the used SiPMs. The multi-clad fibres have a scintillating fibre core with a refractive index of 1.59 surrounded by two claddings with refractive indices of 1.49 and 1.42 , respectively.

\subsection{Quality Control of Scintillating Fibre Parameters}

The attenuation lenght of the fibre material is an important parameter for the light yield of the fibres. Therefore the measurement of this parameter is one of the first quality control checks. The setup, measurement of the fibres and the results are described in [1].

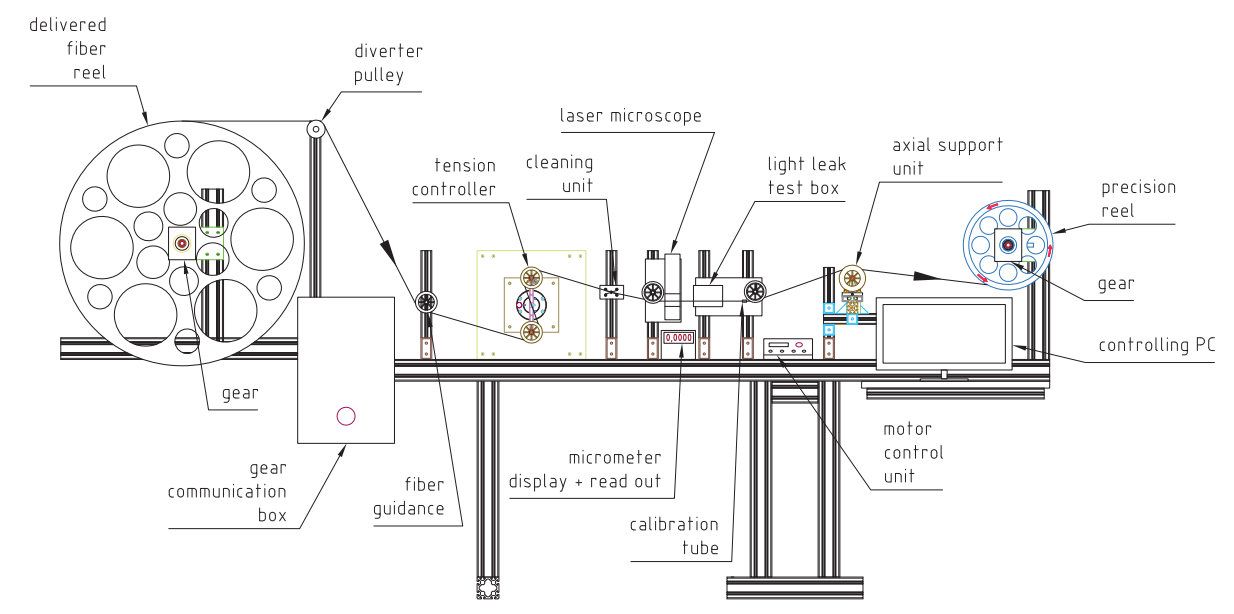

Figure 1: The setup used to rewind and to check the diameter and cladding of the scintillating fibres before fibre mat production

Another parameter which needs to be checked by the quality control is the fibre diameter (nominal $250 \mu \mathrm{m}$ ) and its uniformity. The scintillating fibre arrives on a spool from the producer. These fibres are rewound while measuring the diameter (see fig. 1). The rewinding stand consists of the take-off reel and the take-up reel. Both reels are motor driven and the motor speeds are regulated by a dancer roll tension control unit which ensures a constant tension during the rewindig process. 


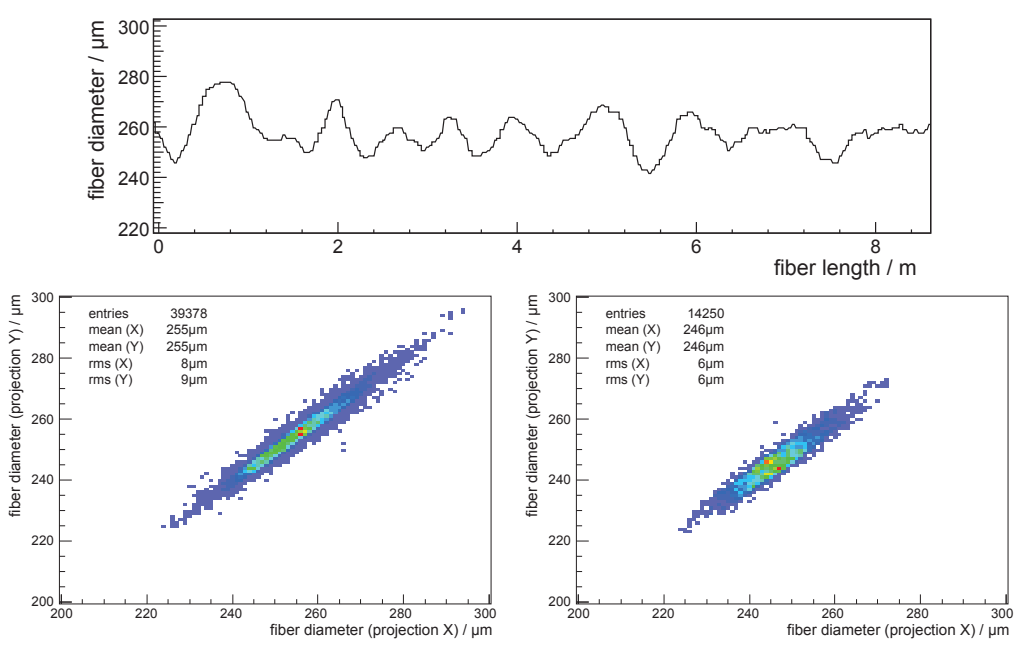

Figure 2: The fibre diameter measurement performed with the 2D-laser microscope. The upper figure shows the variation of the diameter for a piece of SCSF81M fibre plotted against the length. The lower figure shows the correlation of the X-and Y-measured diameter of SCSF81M and SCSF78MJ fibres 吨.

A cleaning device which ensures that the fibre is clean of dust and dirt when it enters the 2D-laser micrometer which measures the fibre diameter. Two orthogonal laser beams are directed from the side onto the object, the shadows of the object are detected by a receiver with a frequency of around $30 \mathrm{~Hz}$, analyzed and the width of the object is calculated. The measured diameter and its variations as a function of fibre length is shown in fig. 2. The diameter of the tested Kuraray SCSF81M fibre was $(255 \pm 8) \mu \mathrm{m}$ and $(246 \pm 6) \mu \mathrm{m}$ for the SCSF78MJ fibre. The correlation between the X-and Y-measurements of 0.98 or 0.93 , respectively, shows that the fibres have a good circularity and are not ellipsoidal (see fig. 2).

After the 2D-laser microscope the fibre passes through a dark box in which $3 \mathrm{SiPMs}$ are mounted. The fibre is excited outside the box by daylight and if the fibre cladding is damaged the SiPMS will detect the changes in the light condition while the fibre is running through the volume.

Before the take-up reel the fibre has to pass through a calibration sleeve which is an injection canula with a diameter of $300 \mu \mathrm{m}$ and acting as a filter. Bulges or knops in the fibre material exceeding the filter limit would cause the fibre to tear.

In front of the take-up reel (winding diameter $314 \mathrm{~mm}$, width $40 \mathrm{~mm}$ ) a pulley is mounted on a motor driven slide which moves the pulley in axial direction by roughly $250 \mu \mathrm{m}$ per revolution of the reel. The reel has a ball bearing mounting on the axle providing low friction easy running when driven by a motor. Brushes are in contact with the fibres during the winding on the reel to avoid electrostatic charging.

\subsection{Production of Scintillating Fibre Mats}

The prepared fibres on the now take-off reel are wound layer by layer on a winding hub guided by a CNC turning lathe. The winding stand is similar to the rewinding stand described in section 2.1. Starting from the take-off reel the fibre is guided via pulleys again through a cleaning device, tension control unit and a tension sensor to the winding hub where the fibre is attached to the hub 
by a bolt. The hub is an aluminum drum which has a helical groove in the surface. The groove has a pitch of $275 \mu \mathrm{m}$ to allow for certain tolerances in the fibre diameter (see fig. 3). After the surface of
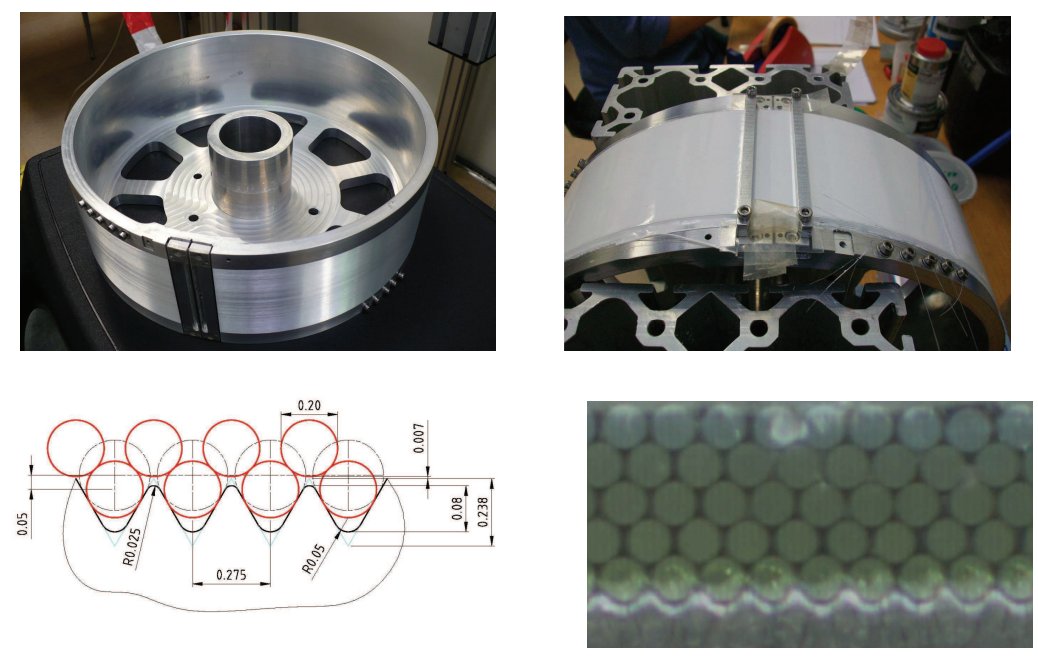

Figure 3: Winding hub (upper left), 5 layer scintillating fiber mat on hub (upper left), scheme of profile of winding grooves (lower left) and photograph of fibers wound into grooves (lower right)

the winding hub is covered with the glue Epotek 301 (optional loaded with $25 \% \mathrm{TiO}_{2}$ to minimize crosstalk between adjacent fibers), the first layer is directly wound onto the hub under a controlled tension of $41.5 \mathrm{cN}$. After each completed layer, the surface is covered again with the Epotek 301 glue and the tension is increased by $\approx 5 \mathrm{cN}$ to compensate for the increasing inner radius of the fibre mat while the fibres of the following layers are wound into the groove-like depressions of the preceding layer. At the end of the winding process of one layer the fibre is fastened with a screw on the opposite margin of the hub and the fibre is cut. Two aluminum end pieces are fixed next to each other on the aluminum drum after the fibre mat winding. The mat is left on the rotating winding hub over night till the glue is cured. The fibre mat is then cut between the end pieces and taken from the winding hub. The end pieces are fixed to a frame, stretching the fibre mat to straighten it. One winding hub with a diameter of $300 \mathrm{~mm}$ and a width of $65 \mathrm{~mm}$ and one hub with a diameter of $840 \mathrm{~mm}$ and a width of $130 \mathrm{~mm}$ have been used to produce fibre mats with a length of $860 \mathrm{~mm}$ or a length of $2650 \mathrm{~mm}$, respectively. The width and the length of the raw mat is larger than needed. Therefore in the remaining last step the final width and length of the mat has to be produced. The mat as a composite material is not easily milled because the fibres at the edges of the mat tend to peel off. Moreover damaged cladding leads to a total loss of the respective fibre. Therefore the milling is done with a high speed cutter made from polycrystalline diamond or alternatively with a carbide blade.

The mechanical carrier supports for the scintillating fibre mats consist of two carbon-fibercomposite (CFC) panels made up from low-density $\left(50 \mathrm{~kg} / \mathrm{m}^{3}\right.$ ) Rohacell foam (thickness 6-10 mm) between two thin (100 mum) carbon fiber skins. The two panels are glued together and a stereo angle of 1 or 2 is ensured (see fig. đ). Polycarbonate end pieces are glued into the module carrier ends. Cutouts were added to the carriers to reduce their mass. The material budget of a complete fibre module including the scintillating fibres is $1.43 \%$ of a radiation length $X_{0}$ or $0.93 \%$ with 

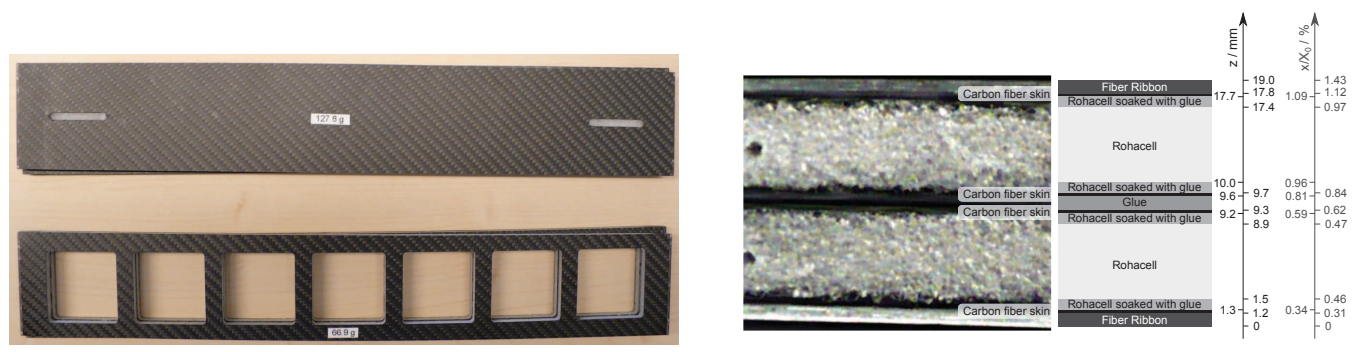

Figure 4: Scintillating fibre module carrier with and without cutouts (left), structure and material budget of a fibre module without cutouts (right)

cutouts. The prepared fibre mat is glued to the module carrier. Its ends are cut and polished in a final step.

The quality control of the scintillating fibre mats is performed optically. The fibre mat is scanned with a standard flatbed scanner. A light source on the remote fibre end is used to inject light into the fibres and the scanner records the opposite end. Broken or damaged fibres which do not transmit the light are detected by their darkness. The position of the fibres is determined by detecting circles. A precise placement of the fibres is desirable since it guarantees a very homogeneous response of the detector. A regular two-dimensional grid is fitted to the positions of the fibre circles. The achieved measured precision for a fibre module was $14 \mu \mathrm{m}$ in the horizontal and $12 \mu \mathrm{m}$ in the vertical direction. The observed precision is similiar in magnitude as the variations in the fibre diameter of $6 \mu \mathrm{m}$ [ 助.
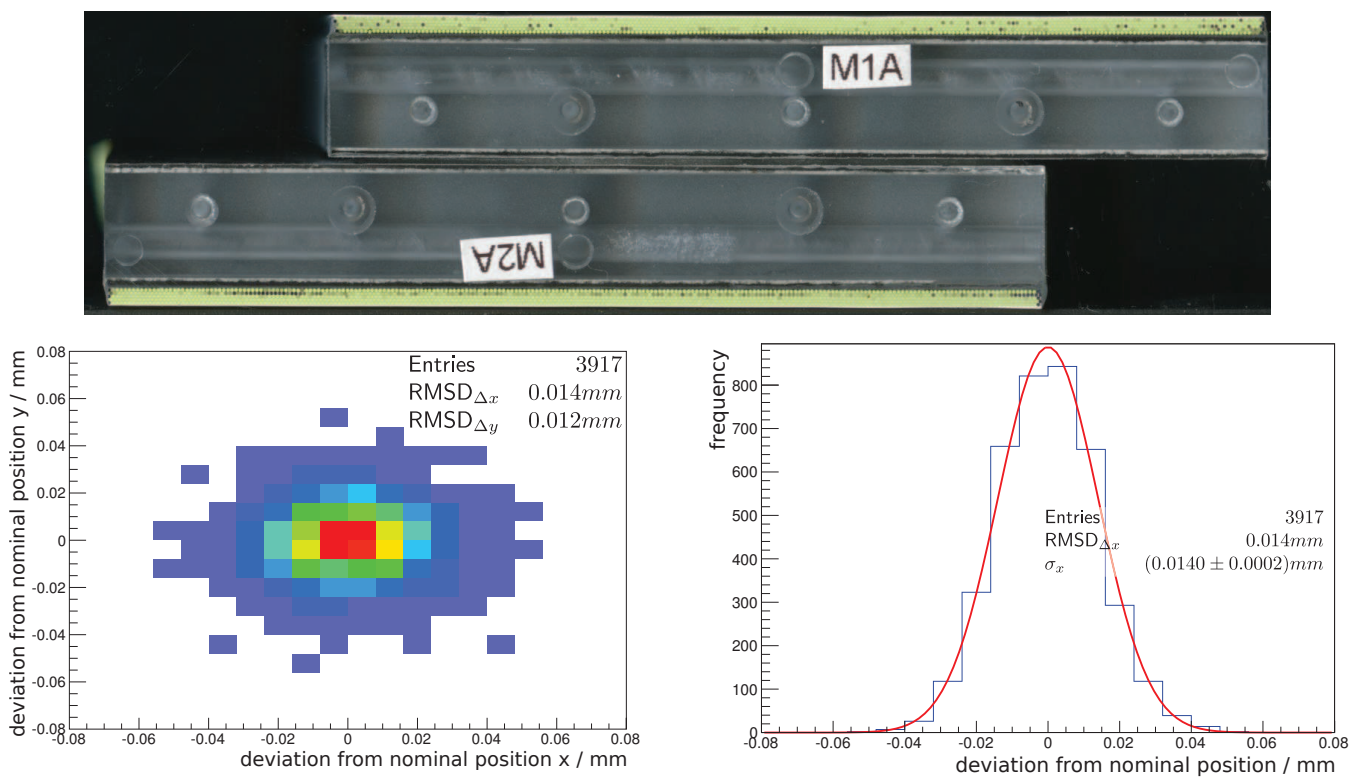

Figure 5: The mechanical precision achieved for modules produced in 2011 using a scan of a module with illumination at the remote fibre end (upper). Deviations from nominal positions in 2D (lower left), projected deviations from nominal positions (lower right) [凹] 


\section{Scintillating Fibre module tracking devices}

\subsection{Prototypes}

Scintillating fibre modules with different types of fibres, SCSF-81M and SCSF-78MJ, were tested in a testbeam at the CERN T9 beamline. Five fibre module prototypes were produced with a length of $860 \mathrm{~mm}$. For the readout the Hamamatsu MPPC 5883 arrays were used with an active area of $8.0 \times 1 \mathrm{~mm}^{2}$ segmented into 32 individual SiPMs with 80 pixels each. The channels have a readout pitch of $250 \mu \mathrm{m}$, matching the fibre diameter. At an overvoltage of $2 \mathrm{~V}$ the MPPCs have a photon detection efficiency of $44 \%$ at $450 \mathrm{~nm}$, a crosstalk probability of $23 \%$, a dark count rate of $200 \mathrm{kHz}$ per channel and breakdown voltage of $70 \mathrm{~V}$ 迎, 2].

The effect of optical grease to couple the SiPM arrays to the fibres was also investigated. A spatial resolution of $50 \mu \mathrm{m}$ and a light yield of 18 photons per minimum ionizing particle was achieved for the modules with Kuraray SCSF-78MJ fibres and optical grease as coupling medium. The setup and results are described in detail in [1], 包].

\subsection{PERDaix scintillating fibre tracker}

The Proton Electron Radiation Detector Aix-la-Chapelle (PERDaix) was launched with the BEXUS-11 balloon flight on 23rd November 2010 and measured 177,000 charged particles during a $1.5 \mathrm{~h}$ long float phase at $33.4 \mathrm{~km}$ altitude. The PERDaix detector consisted of a time-of-flight system, a spectrometer and a transition radiation detector. The spectrometer had a permanent magnet $(B=0.2 \mathrm{~T})$ and a scintillating fibre tracker. It measured the track position on eight points in four double layers. Each double layer consisted of a top and bottom part made of $64 \mathrm{~mm}$ wide, $400 \mathrm{~mm}$ long and $6 \mathrm{~mm}$ thick carbon fibre supported Rohacell foam structures which were glued together and tilted to each other by a relative stereo angle of $1^{\circ}$. Two fibre mats were glued on each side of a module. The Hamamatsu SiPM arrays MPPC 5883 were used again for the readout. Groups of 32 fiber columns were read out by an SiPM array at alternating ends of the module. Two optical hybrids were mounted on each module. Each hybrid held four MPPC 5883 arrays interleaved with four mirrors (see fig. 6). A spatial resolution of $50 \mu \mathrm{m}$ and a light yield of 1620 photons per minimum ionizing particle was achieved during the float phase [[]]. A detailed description of the experiment and the results can be found in [ [
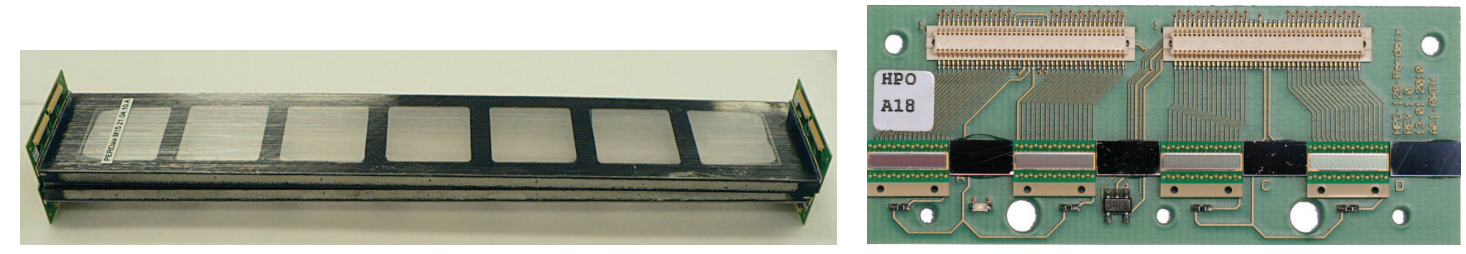

Figure 6: A PERDaix scintillating fibre module (left) and a photo of a PERDaix hybrid showing four MPPC5883 and four mirrors (right).

\subsection{Myontomograph}

A myontomograph is under construction at the I. Physics Institute of RWTH Aachen University and consists of two parts (see fig. ॠ). Between a time of flight system scintillating fibre 
modules are arranged. The upper part has modules with a length of $860 \mathrm{~mm}$ and a width of $64 \mathrm{~mm}$ and a stereo angle of $2^{\circ}$ readout with Hamamatsu SiPM arrays with 128 channels, the lower part is made out of the PERDaix fibre modules with the PERDaix hybrids. A spatial resolution of $50 \mu \mathrm{m}$ is achieved during data taking with cosmic muons. The image of a lead block which was placed between the upper and lower part was reconstructed (see fig. 7) using the tracks of the cosmic muons.
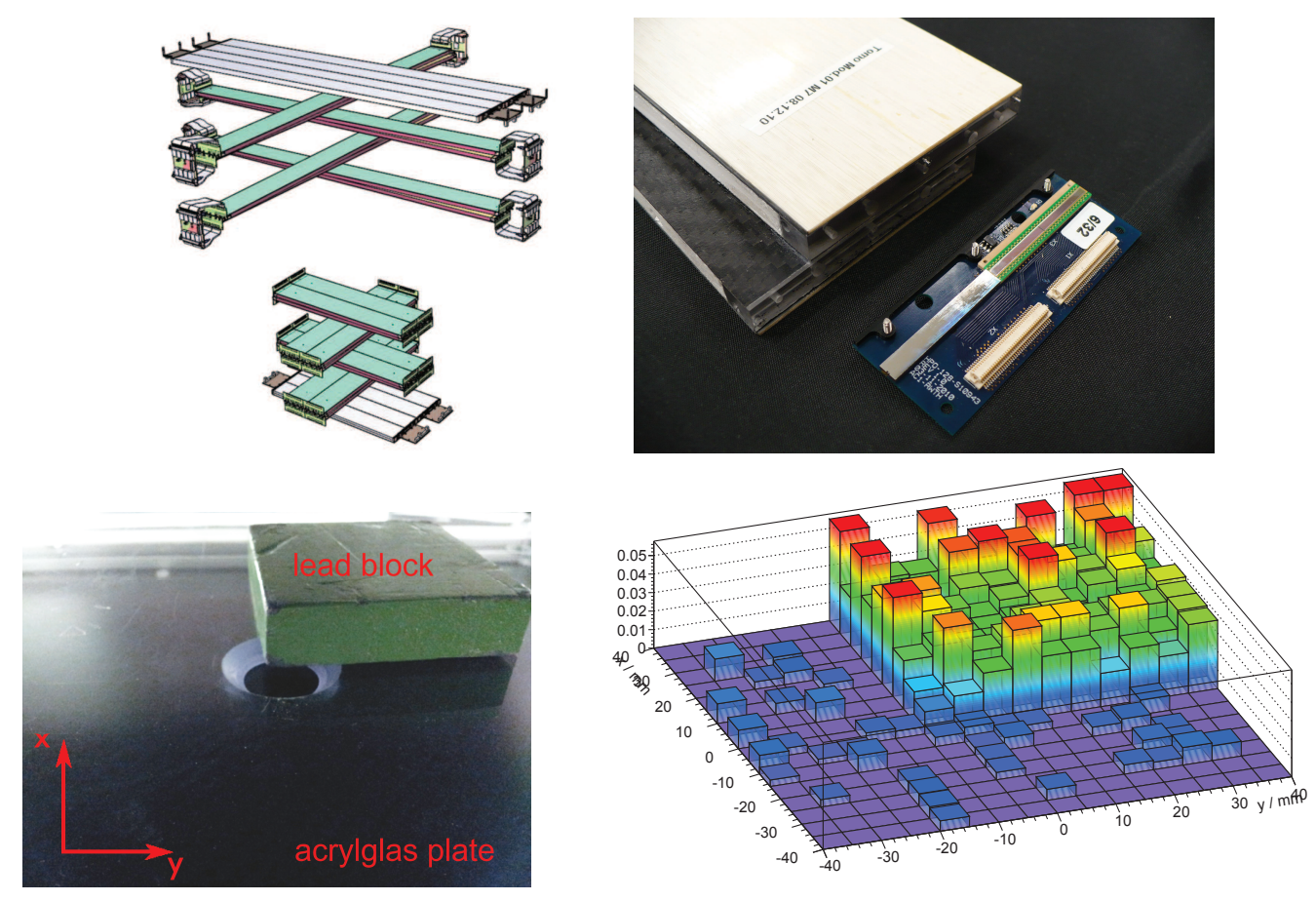

Figure 7: Scheme of a myontomograph with scintillating fibre modules as tracking devices (upper left), a photo of a fibre module and a hybrid showing a 128 channel SiPM array and a mirror (upper right), a lead block placed between upper and lower part of the myontomograph (lower left), reconstructed image of lead block (lower right)

\subsection{Beam Gas Vertex Monitor (BGV)}

The Beam Gas Vertex (BGV) detector is foreseen as a possible non-invasive beam size measurement instrument for the LHC and its luminosity upgrade. The BGV monitor is described elsewhere in this proceedings [5]. This technique is based on the reconstruction of beam-gas interaction vertices, where the charged particles produced in inelastic beam-gas interactions are measured with high-precision tracking detectors.

The BGV demonstrator will have eight scintillating fibre modules which will be arranged around the beam pipe to form two tracking stations near and far from the exit window of the gas injection chamber (see fig 8). The modules of the near station will have four layer fibre mats to minimize the impact of multiple scattering and the ones from the far station will have 5 layer fibre mats. The fibre modules will be readout by SiPM arrays from Hamamatsu and KETEK. 

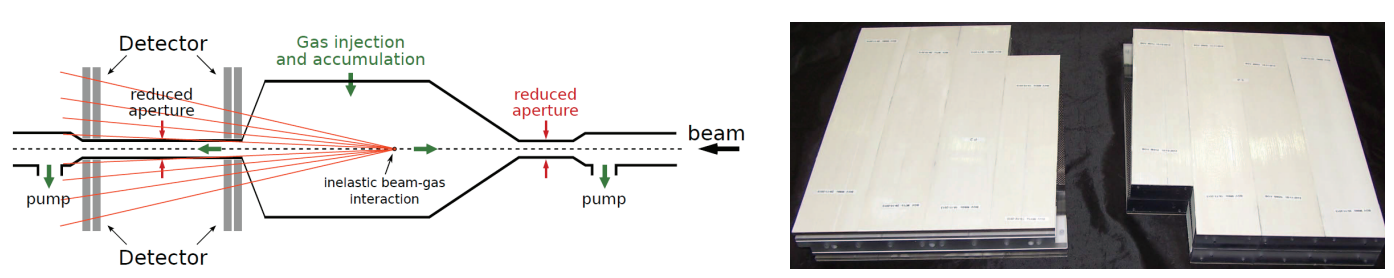

Figure 8: Sketch of the BGV Demonstrator layout (left). The two major components are shown: gas target (chamber for gas injection and accumulation, chambers with reduced aperture and pumping system) and tracking detector, a photo of $2 \mathrm{BGV}$ scintillating fibre module prototypes (right).

\subsection{LHCb Upgrade SciFi Tracker}

The Scintillating Fibre (SciFi) Tracker for the LHCb Upgrade in 2018/19 [6]. The SciFi Tracker is based on $2.5 \mathrm{~m}$ long multi-layered fibre mats from $10000 \mathrm{~km}$ of $250 \mu \mathrm{m}$ diameter scintillating fibres over 12 planes covering $350 \mathrm{~m}^{2}$. For the readout cooled SiPM arrays with 128 channels and $250 \mu \mathrm{m}$ channel width will be used. The LHCb Upgrade SciFi Tracker is described in detail elsewhere in these proceedings [7]. The first 5 layer scintillating fibre mats width a length of $2.5 \mathrm{~m}$ and $3.0 \mathrm{~m}$ and a width of $130 \mathrm{~mm}$ have been produced and are under investigation concerning straightness, light yield and spatial resolution.

\section{References}

[1] B. Beischer et al., The development of a high resolution scintillating fiber tracker with silicon photomultiplier readout, NIM A 628 (2011) 403 - 406

[2] B. Beischer et al., A high-resolution scintillating fiber tracker with silicon photomultiplier array readout,NIM A 622 (2010) 542-554

[3] Th. Kirn et al., The PERDaix detector, NIM A 695 (2012) 91-95

[4] G. Roper, The Development of a High-Resolution Scintillating Fiber Tracker with Silicon Photomultiplier Readout, Ph.D. Thesis, RWTH Aachen University, (2013)

[5] C. Barschel et al., Beam profile measurements based on modern vertex detectors and beam-gas interactions, these proceedings

[6] LHCb collaboration, LHCb Scintillating Fibre Tracker Technical Design Report, CERN-LHCC-2014-001; LHCb TDR 15

[7] B. Leverington et al., The Scintillating Fibre Tracker for the LHCb Upgrade, these proceedings G. Haefeli et al., Silicon Photomultipliers for the LHCb Upgrade Scintillating Fibre Tracker, these proceedings

R. Ekelhof et al., Detector Module Design, Construction and Performance for the LHCb SciFi Tracker, these proceedings 